\title{
Equal Gain Combiner Performance in Equally-Correlated Rayleigh Fading Channels
}

\author{
Yunxia Chen and Chintha Tellambura, Senior Member, IEEE \\ Department of Electrical and Computer Engineering \\ University of Alberta \\ Edmonton, AB, Canada T6G 2V4 \\ Ph:1 7804927228 Fax:1 7804921811 \\ Email: \{yunxia,chintha\}@ece.ualberta.ca
}

\begin{abstract}
This paper develops a novel approach for performance analysis of multi-branch pre-detection equal gain combining (EGC) in equally-correlated Rayleigh fading channels. We convert a set of equally-correlated channel gains to a set of conditionally independent channel gains. The cumulative distribution function (cdf) of EGC output signal-to-noise ratio (SNR) is therefore derived. The symbol error rate (SER) of different modulation schemes with pre-detection EGC in equallycorrelated Rayleigh fading channels is also evaluated. Numerical results that illustrate the effects of equally-correlated fading on the SER performance of EGC are also provided.
\end{abstract}

Key words: Diversity, equal-gain combining, equallycorrelated Rayleigh fading channels, cumulative distribution function

\section{INTRODUCTION}

In recent years, diversity reception (by combining $L$ branch signals) and multi-level modulation schemes have received considerable attention for facilitating high-rate data transmission over wireless links. Pre-detection EGC is of practical interest because it offers performance close to the optimal maximal-ratio combining (MRC) technique but with greater simplicity. However, the literature on the performance analysis of EGC is barren compared with that of other diversity combining schemes. This lack stems from the difficulty of finding the pdf of the EGC output SNR (traditionally, the performance is evaluated by averaging the conditional error probability over the EGC output pdf), which depends on the square of a sum of fading amplitudes. Even for independent fading, a closed-form solution to the pdf of this sum has been elusive (dating back to Lord Rayleigh himself [1]) and indeed, even for the case of Rayleigh fading (mathematically simplest distribution) no solution exists for $L>2$ [2].

Previous studies on independent EGC include the following. Altman and Sichak [3] find the output pdf of dual-branch EGC in Rayleigh fading channels. Due to Beaulieu [2], the pdf of a sum of independent random variables (RV's) can be efficiently approximated. Applying this result, the performances of EGC in Rician and Nakagami fading channels are analyzed [4], [5]. Annamalai et al. [6]-[8] use the Parseval theorem to evaluate the average SER of a broad class of coherent, differentially coherent and non-coherent modulation schemes with predetection EGC.
However, in real-life applications, statistically independent fading can rarely be achieved. For example, in wireless communication handsets, physical constraints may not allow the use of antenna spacing that is required for independent fading across antennas. Thus quantifying the resultant degradation of the performance of diversity systems is a long standing problem of importance [9]. However, for correlated fading channels, all available results on pre-detection EGC deal with dual branches $(L=2)$ (see [10]-[12]). Mallik et al. [10] derive the bit error rate (BER) for dual-branch EGC with coherent detected binary signals in correlated Rayjeigh fading channels. Tellambura and Annamalai [11] derive the characteristic function (chf) of EGC output and utilize an infinite series representation for the complementary error function to obtain the BER. In [12], an integral representation for the Gaussian probability integral is used to derive the BER. To the best of our knowledge, no results have ever been reported for the BER/SER performance of multi-branch $(L>2)$ pre-detection EGC in correlated fading channels. In this paper, we develop a novel approach to analyze the performance of multi-branch pre-detection EGC in equallycorrelated Rayleigh fading channels.

This paper is organized as follows. Section II develops a new representation for equally-correlated Rayleigh channel gains. Section III derives the output cdf of EGC in equallycorrelated Rayleigh fading channels. Section IV evaluates the SER of various digital modulation schemes with pre-detection EGC and also approximate the SER using the Gauss-Laguerre quadrature. Numerical results in Section V illustrate the impact of branch correlation on the performance of pre-detection EGC. Section VI concludes this paper.

\section{Representation of ChanNel Gains}

Rayleigh envelopes are frequently used to model the amplitudes of received signals from urban and suburban areas [13]-[15]. We represent Rayleigh envelopes by using a set of zero-mean complex Gaussian RV's given by

$$
G_{k}=\left(\sqrt{1-\rho} X_{k}+\sqrt{\rho} X_{0}\right)+i\left(\sqrt{1-\rho} Y_{k}+\sqrt{\rho} Y_{0}\right),
$$

for $k=1, \ldots, L$, where $i=\sqrt{-1}, 0 \leq \rho \leq 1, X_{k}$ and $Y_{k},(k=0,1, \cdots, L)$, are two sets of independent zeromean and unit-variance Gaussian RV's. That is, for any $j, k \in$ 
$\{0, \ldots, L\}$

$$
\begin{aligned}
& E\left(X_{k} Y_{j}\right)=0, \\
& E\left(X_{k} X_{j}\right)=E\left(Y_{k} Y_{j}\right)= \begin{cases}1, & k=j, \\
0, & k \neq j .\end{cases}
\end{aligned}
$$

where $E(x)$ denotes the statistical expectation of $x$.

We may write $G_{k}$ in terms of polar coordinates,

$$
G_{k}=R_{k} \exp \left(j \theta_{k}\right), \quad k=1,2, \cdots, L,
$$

where $R_{k}=\left|G_{k}\right|$ is the amplitude of the $G_{k}$. Since $G_{k}$ is a set of complex Gaussian RV's with zero means, $R_{k}$ is a set of Rayleigh envelopes with mean square

$$
E\left(R_{k}^{2}\right)=2 \text {. }
$$

The cross-correlation coefficient between any $G_{k}$ and $G_{j}(k \neq$ $j$ ) equals to $\rho$,

$$
\frac{E\left(G_{k} G_{j}^{*}\right)}{\sqrt{E\left(\left|G_{k}\right|^{2}\right) E\left(\left|G_{j}\right|^{2}\right)}}=\rho, \quad k \neq j .
$$

The relationship between the power correlation (i.e. the correlation between $G_{k}$ and $G_{j}$ ) and envelope correlation (i.e. the correlation between the $R_{k}$ and $R_{j}$ ) is [1, Eq. (1.5-26)],

$$
\rho_{e}=\frac{(1+\rho) E_{i}\left(\frac{2 \sqrt{\rho}}{1+\rho}\right)-\frac{\pi}{2}}{2-\frac{\pi}{2}}
$$

where $E_{i}(\eta)$ denotes the complete elliptic integral of the second kind with modulus $\eta$. For a given $\rho_{e}$, solving (7) yields $\rho$. The solution methods are discussed in [16], [17]. Thus, using (1) and (7), we can readily represent a set of equally-correlated Rayleigh envelopes with a specified value of envelope correlation.

Next, we introduce a 'trick' that will be used in the performance analysis. When $X_{0}=x_{0}$ and $Y_{0}=y_{0}$ are fixed, $G_{k}$ is a set of complex Gaussian RV's with means $\sqrt{\rho}\left(x_{0}+i y_{0}\right)$. Consequently, $R_{k}$ is a set of identically and independent distributed (iid) Rician RV's with Rician factor $\rho\left(x_{0}^{2}+y_{0}^{2}\right) / 2 /(1-\rho)$. Performance analysis can now be carried out in two steps. First, we evaluate the conditional performance of EGC for a given $x_{0}^{2}+y_{0}^{2}$. Second, we average the conditional results over the distribution of $X_{0}^{2}+Y_{0}^{2}$.

\section{DERIVATION OF EGC OUTPUT CDF}

Using the new representation for the channel gains (1), we derive the expression for the cdf of balanced branch EGC output SNR in equally-correlated Rayleigh fading channel. We assume the receive signals at different branches to be identically distributed and equally-correlated with each other. The noise components at different branches are assumed to be independent of the signal components and uncorrelated with each other.
In pre-detection EGC, received signals are first co-phased and then summed to form the resultant output. The instantaneous SNR at the output can be written as

$$
\gamma_{\text {egc }}=\frac{\left(R_{1}+R_{2}+\cdots+R_{L}\right)^{2} E_{s}}{L N_{0}}
$$

where $L$ denotes the diversity order, $N_{0}$ is the noise power spectral density per branch and $E_{s}$ is the energy of transmitted signal.

We consider $X_{0}$ and $Y_{0}$ to be fixed $\left(X_{0}^{2}+Y_{0}^{2}=U\right)$. Then $R=R_{1}+R_{2}+\cdots+R_{L}$ is a sum of $L$ iid Rician RV's with Rice factor and average power given by

$$
\begin{gathered}
K=\frac{\rho u}{2(1-\rho)}, \\
\Omega=2(1-\rho)+\rho u .
\end{gathered}
$$

Evaluating the output cdf for a fixed $x_{0}^{2}+y_{0}^{2}=u$, we obtain the conditional output cdf,

$$
\begin{aligned}
F_{\gamma_{c g c}}\left(\left.y\right|_{x_{0}^{2}+y_{0}^{2}=u}\right) & =\operatorname{Pr}\left(\frac{r^{2} E_{s}}{L N_{0}} \leq\left. y\right|_{x_{0}^{2}+y_{0}^{2}=u}\right) \\
& =\operatorname{Pr}\left(r \leq\left.\sqrt{\frac{L N_{0 y} y}{E_{s}}}\right|_{x_{0}^{2}+y_{0}^{2}=u}\right) \\
& =F_{R}\left(\left.\sqrt{\frac{2 L y}{\tilde{\gamma}}}\right|_{x_{0}^{2}+y_{0}^{2}=u}\right) .
\end{aligned}
$$

where $\bar{\gamma}=\frac{E_{s}}{N_{0}} E\left(R_{k}^{2}\right)=\frac{2 E_{s}}{N_{0}}$ is the average branch SNR, and $F_{R}(x)$ is the cdf of $R$ which can be computed using a convergent infinite series [2]. The computation for $F_{R}(x)$ has been discussed in detail [5, Eq. $(9,14,15)]$. For brevity, we omitted those formulas here.

Notice that $X_{0}^{2}+Y_{0}^{2}$ is chi-square distributed with two degrees of freedom and its pdf can be found [18]

$$
p(u)=\frac{1}{2} e^{-u / 2}, \quad u \geq 0 .
$$

Averaging the conditional cdf (11) over the distribution of $U$, we finally obtain the cdf for multi-branch EGC in equally correlated Rayleigh fading channel,

$$
F_{\gamma_{\text {eqc }}}(y)=\frac{1}{2} \int_{0}^{\infty} F_{R}\left(\left.\sqrt{\frac{2 L y}{\bar{\gamma}}}\right|_{x_{0}^{2}+y_{0}^{2}=u}\right) e^{-u / 2} d u .
$$

This is a novel result which enables the evaluation of the outage of multi-branch EGC systems in equally-correlated Rayleigh fading channels.

Using variable substitution $x=u / 2$ and then applying Gauss-Laguerre quadrature, we obtain a series expression for (13)

$$
F_{\gamma_{\text {esc }}}(y) \approx \sum_{m=1}^{M} w_{m} F_{R}\left(\left.\sqrt{\frac{2 L y}{\bar{\gamma}}}\right|_{x_{0}^{2}+y_{0}^{2}=2 x_{m}}\right)
$$


where $x_{m}$ is the $m$-th zero of Laguerre polynomials $L_{M}(x)$ and $w_{m}$ is the corresponding weight factor given by [19]

$$
\omega_{m}=\frac{(M !)^{2} x_{m}}{(M+1)^{2}\left[L_{M+1}\left(x_{m}\right)\right]^{2}} .
$$

Tables for $x_{m}$ and $\omega_{m}$ can be found in [19], [20].

\section{Performance Analysis}

Following the same approach as the above, we may evaluate the average SER of various digital modulation schemes with pre-detection EGC in equally-correlated Rayleigh fading channels. We assume that channel fading is nonselective and changing slowly enough so that the channel parameters remain constant for the duration of signaling interval.

Recall that when $X_{0}$ and $Y_{0}$ are fixed $\left(X_{0}^{2}+Y_{0}^{2}=U\right), R_{k}$ is a set of iid Rician RV's with Rician factor given by (9). Due to Annamalai et al. [7], we can readily obtain the conditional SER for a fixed $x_{0}^{2}+y_{0}^{2}=u$

$$
P_{s}\left(\left.\bar{\gamma}\right|_{x_{0}^{2}+y_{0}^{2}=u}\right)=\frac{2}{\pi} \int_{0}^{\pi / 2} \frac{\Psi\left(\left.\tan \zeta\right|_{x_{0}^{2}+y_{0}^{2}=u}\right)}{\sin (2 \zeta)} d \zeta
$$

where $\Psi(\omega)=\operatorname{Real}\left[\omega G(\omega) \phi_{r}^{*}(\omega)\right]$ and where $\operatorname{Real}(x)$ denotes the real part of $x$, * denotes conjugate operation. $G(\omega)$ is the Fourier transform (FT) of generic conditional error probability which can be found in [7] and $\phi_{r}(\omega)$ is the chf of the square root of output SNR $\left(\sqrt{\gamma_{\text {egc }}}\right)$ given by [7]

$$
\begin{aligned}
& \phi_{r}\left(\left.\omega\right|_{x_{0}^{2}+y_{0}^{2}=u}\right) \\
& =\left[\frac{e^{-\frac{\rho u}{2 \pi-\rho)}}}{\pi} \int_{0}^{\pi} \exp \left(\left(j \frac{\omega \xi}{2}+\sqrt{\frac{\rho u}{1-\rho}} \frac{\cos \theta}{2}\right)^{2}\right)\right. \\
& \left.\times D_{-2}\left(-j \omega \xi-\sqrt{\frac{\rho u}{1-\rho}} \cos \theta\right) d \theta\right]^{L}
\end{aligned}
$$

where $j=\sqrt{-1}, \xi=\sqrt{\frac{(1-\rho) \bar{\gamma}}{2 L}}$ and $D_{-2}(x)$ is parabolic cylinder function which is defined as [21, Eq. (9.240)].

Averaging the conditional SER (16) over the distribution of $U$ (12), we obtain the average SER as

$$
P_{s}(\bar{\gamma})=\frac{1}{\pi} \int_{0}^{\infty} \int_{0}^{\pi / 2} \frac{\Psi\left(\left.\tan \zeta\right|_{x_{0}^{2}+y_{0}^{2}=u}\right)}{\sin (2 \zeta)} d \zeta e^{-u / 2} d u .
$$

Therefore, we may evaluate the average SER of various modulation schemes with pre-detection EGC using [7, Eq. $(11,13,16,17,20)]$ and (17) with (18). For example, the SER of differential binary phase-shift-keying (DPSK) can be obtained as

$$
\begin{aligned}
P_{s}(\bar{\gamma}) & =\frac{1}{\pi} \int_{0}^{\infty} \int_{0}^{\pi / 2} \operatorname{Real}\left\{\frac { \operatorname { t a n } \zeta } { 2 } \left[\frac{\sqrt{\pi}}{2} \exp \left(-\frac{\tan ^{2} \zeta}{4}\right)\right.\right. \\
& \left.\left.+j F\left(\frac{\tan \zeta}{2}\right)\right] \phi_{r}^{*}\left(\left.\tan \zeta\right|_{x_{0}^{2}+y_{0}^{2}=u}\right)\right\} \frac{e^{-u / 2}}{\sin (2 \zeta)} d \zeta d u
\end{aligned}
$$

where $F(x)$ denotes the Dawson integral [22].

Annamalai et al. [7] derive an approximation for $P_{s}\left(\left.\bar{\gamma}\right|_{x_{0}^{2}+y_{0}^{2}=u}\right)$ using Gauss-Chebychev quadrature. Based on their results and applying Gauss-Laguerre quadrature, we approximate the average SER as

$$
P_{s}(\bar{\gamma}) \approx \frac{1}{N_{1}} \sum_{m=1}^{M} w_{m} \sum_{n=1}^{N_{1}} \frac{\Psi\left[\left.\frac{1}{\sqrt{2}} \tan \left(\frac{(2 n-1) \pi}{4 N_{1}}\right)\right|_{\left.x_{0}^{2}+y_{0}^{2}=2 x_{m}\right]}\right.}{\sin \left(\frac{(2 n-1) \pi}{2 N_{1}}\right)}
$$

where $x_{m}$ and $w_{m}$ are defined as (15) and $\phi_{r}\left(\left.\omega\right|_{x_{0}^{2}+y_{0}^{2}=2 x_{m}}\right)$ can be approximated as [23]

$$
\begin{aligned}
& \phi_{r}\left(\left.\omega\right|_{x_{0}^{2}+y_{0}^{2}=2 x_{m}}\right) \\
& \approx\left[\frac{e^{-\frac{\rho x_{m}}{(1-\rho)}}}{N_{2}} \sum_{n=1}^{N_{2}} \exp \left(\left(j \frac{\omega \xi}{2}+\sqrt{\frac{2 \rho x_{m}}{1-\rho}} \frac{\cos \theta_{n}}{2}\right)^{2}\right)\right. \\
& \left.\times D_{-2}\left(-j \omega \xi-\sqrt{\frac{2 \rho x_{m}}{1-\rho}} \cos \theta_{n}\right)\right]^{L}
\end{aligned}
$$

where $\theta_{n}=\frac{(2 n-1) \pi}{2 N_{2}}$. There is a tradeoff involved in the choice of $N_{1}, N_{2}$ and $M$. A greater accuracy may be obtained using larger values, but at the expense of increasing computation complexity.

\section{NUMERICAL RESULTS}

Numerical results are next given to illustrate the effect of correlation on the performance of pre-detection EGC in equally-correlated Rayleigh fading channels. In all the figures, $\rho$ is the power correlation coefficient and $\bar{\gamma}$ is the average branch SNR.

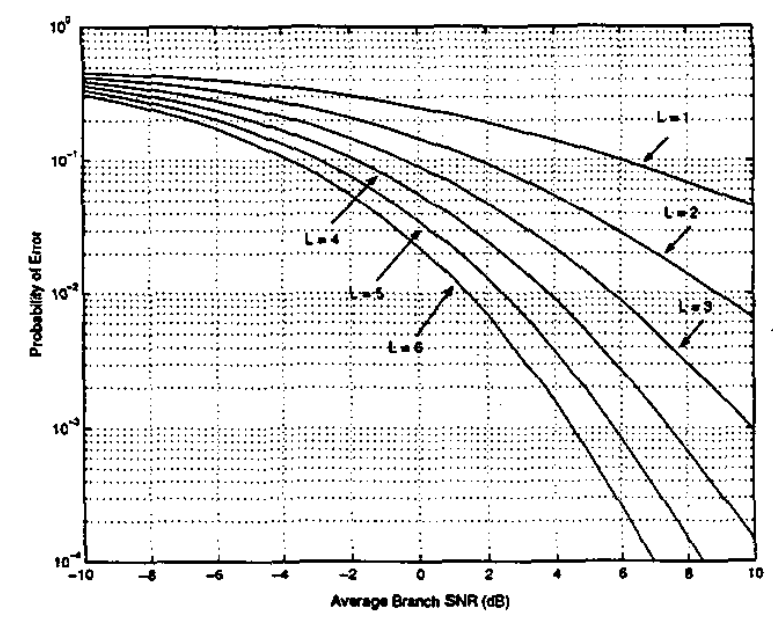

Fig. 1. Average error rate of DPSK with pre-detection EGC in equallycorrelated Rayleigh fading channels; $(\rho=0.5)$.

Fig. 1 shows the effect of diversity order $L$ on the performance of DPSK with pre-detection EGC in equally-correlated Rayleigh fading channels with $\rho=0.5$. The case of $L=1$ represents a situation with no diversity. As expected, diversity gain can still be achieved even with correlated fading. The 
maximum diversity gain is achieved with dual-branch diversity. With increasing of $L$, diversity gain diminishes. This is more pronounced at a higher SNR level.

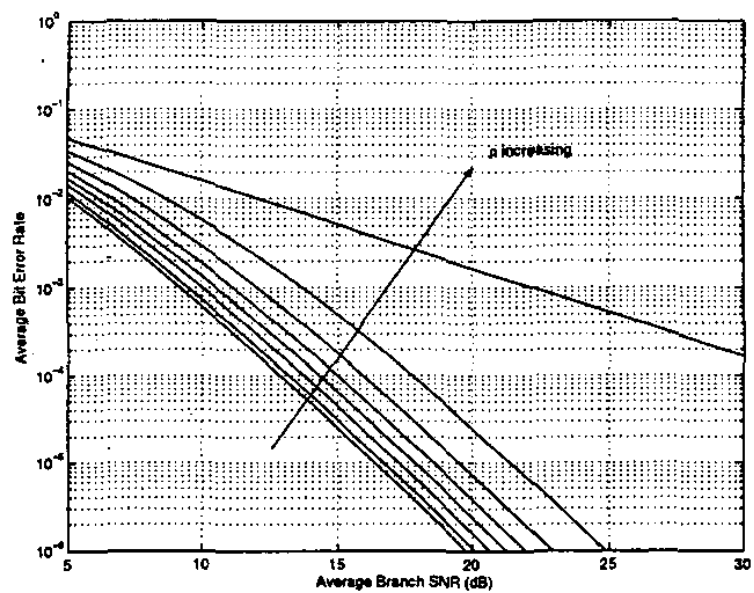

Fig. 2. Average error rate of DPSK with triple-branch pre-detection EGC in equally-correlated Rayleigh fading channels: $(L=3, \rho=$ $0.0 .3,0.5,0.6 .0 .7 .0 .8,0.9,1)$. Circles denote simulation results

Figs. 2 shows the effect of $\rho$ on the performance of DPSK with triple-branch pre-detection EGC in equally-correlated Rayleigh fading channels. The case of $\rho=0$ represents independent fading. Observe that the correlation between the branch signals results in large loss in performance. The diversity gain decreases as $\rho$ increases.

\section{CONCLUSION}

We have developed a new representation for equallycorrelated Rayleigh channel gains. We showed that this representation enables the performance analysis of pre-detection EGC in such channels. Numerical results show that diversity gains can still be achieved in correlated Rayleigh fading channels.

The representation developed in this paper can also be used to analyze the performance of multi-branch SC and generalized SC schemes in equally-correlated Rayleigh fading channels. These results will be reported in a forthcoming paper.

\section{ACKNOWLEDGEMENT}

This work has been supported in part by NSERC and iCORE.

\section{REFERENCES}

[1] W. C. Jakes, Micnowave Mobile Communications. IEEE Press, 1994.

[2] N. C. Beaulieu, "An infinite series for the computation of the complementary probability distribution function of a sum of independent randorn variables and its application to the sum of Rayleigh random variables," IEEE Trans. Commun., vol. 38, no. 9, pp. 1463-1474, Sept. 1990.

[3] F. J. Altman and W. Sichak. "A simplified diversity communication system for beyond the horizon links." IRE Trans. Commun. Sy.st., vol. 4. pp. S0-55, Mar. 1956
[4] N. C. Beaulieu and A. A. Abu-Dayya, "Analysis of equal gain diversity on Nakagami fading channels," IEEE Trans. Commun., vol. 39, no. 2. pp. 225-234, Feb. 1991.

[5] A. Abu-Dayya and N. C. Beaulieu, "Microdiversity on Ricean channels," IEEE Trans. Commun., vol. 42, pp. 2258-2267, June 1994.

[6] A. Annamalai, C. Tellambura, and V. K. Bhargava, "Exact evaluation of maximal-ratio and equal-gain diversity receivers for M-ary QAM on Nakagami fading channels," IEEE Trans. Commun., vol. 47, pp. 13351344, Sept. 1999.

[7] A. Annamalai, C. Tellambura, and V. Bhargava. "Unified analysis of equal-gain diversity on Rician and Nakagami fading channels." in Wireless Communications and Networking Conference. IEEE. 1999. pp. 10-14.

[8] A. Annamalai, C. Tellambura and V.K. Bhargava, "Equal-gain diversity receiver performance in wireless channels," vol. 48, pp. 1732-1745, Oct. 2000.

[9] J. N. Pierce and S. Stein, "Multiple diversity with nonindependent fading," Proc. IRE, vol. 48, pp. 196-211, Oct. 1960.

[10] R. Mallik, M. Win, and J. Winters, "Performance of predetection dual diversity in correlated rayleigh fading: EGC and SD," in IEEE GLOBECOM. IEEE, 2000, pp. 932-936.

[11] C. Tellanbura and A. Annamalai, Wiley Encyclopedia of Telecommunicutions. Wiley, Jan 2003, ch. Wireless Communications Systems Design.

[12] A. Annamalai, V. Ramanathan and C. Tellambura, "Analysis of equalgain diversity receiver in correlated fading channels," Vehicular Technology Conference, 2002, vol. 4, pp. 2038 -2041, 2002.

[13] W. R. Young, "Comparison of mobile radio transmission at 150, 450, 900 , and $3700 \mathrm{MHz}, "$ Bell Sys. Tech. J., vol. 31, pp. 1068-1085, 1952.

[14] H. W. Nylund. "Characteristics of smalt-area signal fading on mobile circuits in the $150 \mathrm{MHz}$ band," vol. 17, pp. 24-30, Oct. 1968.

[15] Y. Okumura, E. Ohmori, T. Kawano and K. Fukuda. "Field strength and its variability in VHF and UHF land mobile radio services," Rev. Elec. Commun. Lab., vol. 16, pp. 825-873, Sept/Oct. 1968.

[16] R. B. Ertel and J. H. Reed, "Generation of two equal power correlated Rayleigh fading envelopes," IEEE Commun. Lets., vol. 2, no. 10, pp. 276-278, 1998.

[17] N. C. Beaulieu, "Generation of correlated Rayliegh fading envelopes," IEEE Commun. Lett., vol. 3, no. 6, pp. 172-174, June 1999.

[18] J. G. Proakis, Digizal Communications, 3rd ed., ser. McGraw-Hill. New York: McGraw-Hill, 1995.

[19] M. Abramowitz and I. A. Stegun. Handhook of Mathematical Functions with Formulas, Graphs, and Mathematical Tables: New York: Dover, 1972.

[20] H. E. Salzer and R. Zucker, "Table of the zeros and weight factors of the first fifteen laguerre polynomials," Bull. Amer. Math. Soc., vol. 55. pp. $1004-1012,1949$.

[21] I. S. Gradshteyn and I. M. Ryzhik, Table of Integrals, Series, and Products, 5th ed. Academic Press. Inc.. 1994.

[22] G. B. Rybicki. "Dawson's integral and the sampling theorem." Computers in Physics, vol. 3, no. 2, pp. 85-87. Mar. 1989.

[23] A. Annamalai. C. Tellambura, and V. K. Bhargava. "A simple and accurate analysis of digital communication systems with diversity reception in different fading environments," in IEEE PIMRC. IEEE, 1998, pp. $1055-1060$. 